\title{
Recent Research and Development of Microgrids in Parana
}

\author{
Mariana Machado ${ }^{1}$ \\ https://orcid.org/0000-0003-1134-8785
}

Vanderlei Aparecido Silva ${ }^{1}$

https://orcid.org/0000-0001-8253-1179

Thaís Marzalek Blasi ${ }^{1}$

https://orcid.org/0000-0002-8933-1521

\author{
Alexandre Rasi Aoki ${ }^{1 *}$ \\ https://orcid.org/0000-0001-9863-6610
}

Thelma Solange Piazza Fernandes ${ }^{1}$

https://orcid.org/0000-0002-5167-1547

Germano Lambert-Torres ${ }^{2}$

https://orcid.org/0000-0003-3789-4696

\section{Kristie Kaminski Küster ${ }^{1}$}

https://orcid.org/0000-0001-5736-0072

${ }^{1}$ Federal University of Parana, Department of Electrical Engineering, Curitiba, PR, Brazil; ${ }^{2}$ Gnarus Institute, R\&D Department, Itajuba, MG, Brazil.

Editor-in-Chief: Paulo Vitor Farago

Associate Editor: Fabio Alessandro Guerra

Received: 2021.03.24; Accepted: 2021.04.07.

*Correspondence: aoki@ufpr.br; Tel.: +55-41-32086334 (A.R.A.).

\section{HIGHLIGHTS}

- Efforts in research to enable a microgrid-based power system infrastructure are shown.

- In a first level, by the optimization of the assets within a microgrid via multi-objective optimization;

- In a second level, by the optimization of microgrid while connected to the main grid via OPF analysis;

- In a third level, by the optimization of multi-microgrids connected together and to the grid.

- Alternatively, by considering decentralized energy markets as an integration approach.

Abstract: Microgrids (MGs) have increasingly attracted the attention of researchers, government officials, and electricity companies for the values and services they can add to the grid. They can operate independently as a single controllable unit in a coordinated way while connected or islanded, which make them suitable to integrate the growing amount of distributed energy resources (DERs) into the distribution network (DN). An active distribution network with high penetration of DERs could be redefined into a collection of microgrids in different layers, pooling their resources together to enhance the grid's performance in the sense of electricity as a service. However, enabling a microgrid-based power system infrastructure poses challenges concerning operation and control. In this paper, a comprehensive overview of research topics regarding microgrid operation are shown in a hierarchical or stratified manner, looking for a holistic vision of a microgrid-based distribution system. First, multi-objective optimization within one microgrid is formulated aiming to minimize costs and maximize battery life is presented. Second, the interaction among MGs, DERs and the DN was modeled using the OPF technique to represent the operation of the grid as a whole. 
Following, a methodology for collaborative optimization of multiple MGs operating together is presented and at last a theoretical framework for MG operation through decentralized energy markets is discussed. As a result, this paper aims to contribute to a broad vision of the distribution system based on microgrid operation in all its levels.

Keywords: Microgrid; Active distribution network; Multi-microgrid; Transactive energy.

\section{INTRODUCTION}

Over the past 15 years, microgrids (MG) have increasingly attracted the attention of researchers, government officials, and electricity companies for the values and services they can add to the grid. In 2011, the Microgrid Exchange Group defined a microgrid as a group of interconnected loads and distributed energy resources (DER) with clearly defined electrical boundaries, which can act as a single and controllable entity in the main grid, with the ability to operate in both grid-connected and island mode [1].

According to this definition, microgrids could be seen as modular pieces, that, connected together and arranged hierarchically, form the distribution system infrastructure. In other words, the electric grid itself could be viewed as a smart super-structure of microgrids synchronized together. This concept would serve a decentralized view of energy production and consumption, associated with integration of renewable energy sources (RES), reduction of greenhouse gases, resilience, and reliability. All of them are well-known advantages that an MG can offer [2,3].

However, a distribution network based on MG and DER can present new challenges concerning operation and control. The effects of an increased level of penetration of RES and the coordination of multiple energy resources throughout the distribution network, such as distributed generation (DG), storage systems, and flexible loads, are examples of new issues to the grid operation. Thus, there is a growing interest in recent researches to investigate how to coordinate and manage all these new features in a distribution network [4] while meeting the 3D's transformation process based on decarbonization, decentralization, and digitalization.

There is an increasing interest in MG development in Brazil. In the last decade, the Brazilian Electricity Regulatory Agency (named ANEEL in Portuguese) developed several public calls for strategic research and development (R\&D) projects within topics of DER integration such as photovoltaic energy, energy storage systems and electrical mobility. Attuned to ANEEL's efforts, the power distribution company of the State of Paraná, Copel Distribuição S.A. is developing R\&D projects for optimizing DERs into the distribution grid, evolving it to an active distribution grid. Copel aims at developing a leading smart grid operational center and reaching expertise in MG technology. By the end of 2020, this utility released the DIS GD Public Call 001/2020 (public notice of widespread disclosure for contracting public works and services without bidding, according to federal law) for contracting energy from microgrids [5], with deadline for receipt of proposals by June 2021. The initiative aims to offer incentives to energy producers who want to build MGs in Copel's DN and thus contribute to improving the company's reliability indicators. As a result, the State of Parana may soon have several MGs in its DN.

This work presents a compendium of study on MG that help set the path to a vision of a microgrid-based distribution system. Each section addresses a specific topic, which represents a research segment being carried out in the State of Paraná on the subject of MG, with a brief literature review followed by a subject development, and simulation results when applicable. Each section should be viewed as a study in itself regarding its specific theme. They share an overlapping relationship, therefore exploring the MG operation topics cohesively from a single MG unit to multiple MG and advanced energy markets. By bringing them together, this paper aims to highlight the issues for a broad vision of an MG based distribution system including technological framework, planning and optimization of operation, MG integration to the active distribution network, multi-microgrid operation, and market issues.

The optimization of a single microgrid has been widely studied in the literature [6-9], as MG needs to be economically efficient, producing energy at the lowest possible cost while eliminating or minimizing losses, maximizing their assets' useful life, and meeting operational constraints. In the present work, a multi-objective optimization problem of a microgrid is formulated in the second section. The objectives are to minimize costs and maximize battery life considering DG, an energy storage system (ESS), and residential loads.

However, an investigation on the integration between MG and the active distribution network is also necessary as MG usually operates in the grid-connected mode. Therefore, the Distribution System Operator (DSO) needs to verify the impacts that MG and DER can cause in the operation of a distribution network and then establish the operational limits to be met by them. Some works have investigated this subject using 
optimal power flow (OPF) as in $[10,11]$. In the third section, the present work seeks to investigate the interaction among MG, DER, and the distribution network using the OPF technique to model the operation of the grid as a whole.

Furthermore, with the increasing popularity of MGs, some areas of the distribution network may contain several MG, each with its purposes of operation, which may not necessarily be the same purposes as the DSO. Also, an independent optimization of the MGs can generate conflicting results. Thus, it is necessary to have an optimization methodology that takes into account the interests of the various stakeholders, seeking an optimal collaborative result for the group as in [12-14], which is characterized as multi-microgrid (MMG) optimization. The fourth section of the present work addresses the optimization of MMG, presents a short literature review with the main works published, explores MMG concepts, and presents a methodology for collaborative optimization of multiple MG.

Finally, the fifth section addresses MG from the point of view of decentralized energy markets, where the network operation is transaction-driven. This concept represents a new paradigm for future energy markets. After a short bibliographic review, a theoretical framework is presented, and at the end of the section, further developments are discussed.

\section{MICROGRID OPTIMIZATION}

Proper microgrid energy management enables it to operate within technical specifications while meeting operational objectives such as minimize operation costs and pollutant emissions or maximize battery life $[8,15,16]$. Optimization tools can be used to determine the optimal operating points of the system that can be used to plan the operation of a microgrid. Previous studies were developed to solve these issues. However, their main focus is on isolated microgrid optimization instead of a grid-connected microgrid.

The present section aims to develop a multi-objective optimization of a microgrid based on the NonDominated Sorting Genetic Algorithm II (NSGA-II) to minimize costs and maximize battery life. Local elements in the model were considered such as tariffs, generation type, distribution system characteristics, and costs. In this section, the microgrid is comprised of photovoltaics, batteries, and residential loads.

The first part that composes the multi-objective function is presented at equation (1) which is the criterion that minimizes operation costs [6]:

$$
\min \text { costs }=\min \sum_{t=1}^{N} \sum_{i=1}^{M}\left(P_{p v}^{i, t} \cdot c_{p v}^{i}+P_{\text {charge }}^{i, t} \cdot c_{\text {charge }}^{i}+P_{\text {discharge }}^{i, t} \cdot c_{\text {discharge }}^{i}+P_{\text {purchase }}^{i, t} \cdot c_{\text {purchase }}^{i}-P_{\text {sell }}^{i, t} \cdot c_{\text {sell }}^{i}\right)
$$

where $P_{p v}^{i, t}$ is the power provided by photovoltaics, $P_{\text {charge }}^{i, t}$ is the power charged by batteries; $P_{\text {discharge }}^{i, t}$ is the power discharged by batteries, $P_{\text {purchase }}^{i, t}$ is the power purchased from the distribution grid, $P_{\text {sell }}^{i, t}$ is the power sold for the distribution grid, all of them for instant $t$ and element $i ; c_{p v}$ is the photovoltaic operation and maintenance costs $[\$ / \mathrm{kWh}], c_{\text {charge }}$ is the battery charge cost $[\$ / \mathrm{kWh}], c_{\text {discharge }}$ is the battery discharge cost $[\$ / \mathrm{kWh}], c_{\text {purchase }}$ is the purchase price of energy $[\$ / \mathrm{kWh}]$ and $c_{\text {sell }}$ is the selling price of energy $[\$ / \mathrm{kWh}]$.

The second part that composes the multi-objective function is presented at equation (2) which is a function that maximizes battery life, to be calculated through minimization of battery degradation [17]:

$$
\min \text { degradation }=\min D_{\text {TOTAL }}
$$

The model degradation equations were based on [17], where total battery degradation is calendar degradation plus cycling degradation, as presented in equation (3).

$$
D_{\text {TOTAL }}=D_{\text {calendar }}+D_{\text {cycling }}
$$

where $D_{\text {TотАL }}$ is the total battery degradation, $D_{\text {calendar }}$ the calendar degradation, and $D_{\text {cycling }}$ cycling degradation.

Calendar degradation is represented by equation (4).

$$
D_{\text {calendar }}=\frac{t_{t}}{t_{f}} \cdot 2^{\frac{T-25}{10}}
$$

where $t_{t}$ is the period since battery manufacturing, $t_{f}$ is the calendar life informed by the manufacturer, and $T$ is the battery temperature in degrees Celsius. 
On the other hand, cycling degradation is obtained by equation (5).

$$
D_{\text {cycling }}=\sum_{i=1}^{n \text { cycles }} D_{\text {cycling }}\left(25^{\circ} \mathrm{C}\right)\left(D_{o} D_{i}\right) \cdot 2^{\frac{T-25}{10}}
$$

where $D_{\text {cycling }}$ is the cycling degradation at temperature $T\left({ }^{\circ} \mathrm{C}\right), D_{\text {cycling }}\left(25^{\circ} \mathrm{C}\right)$ the cycling degradation at $25^{\circ} \mathrm{C}, T$ is the battery temperature in degrees Celsius, and $D_{o} D_{i}$ is the corresponding value of degradation obtained through the cycle curve versus the depth of discharge.

Therefore, the multi-objective function is presented by equation (6) is conditioned by constraint equations given by (7)-(15) [6], and referred to each of the 24 hours of planning. Inequalities (7)-(9) represent the operational limits whereas equation (10) shows the active power balance. Moreover, inequalities (11)-(14) express battery operational limits, and equation (15) represents the state of charge at instant $t+1$.

$$
\begin{gathered}
\text { multi-objective function }=(\min \text { costs })+(\text { min degradation }) \\
\qquad P_{i}^{\text {min }} \leq P_{i}^{t} \leq P_{i}^{\text {max }}
\end{gathered}
$$

where $P_{i}^{\min }$ is the minimum power generation and $P_{i}^{\max }$ is the maximum power generation.

$$
P_{i j}^{\min } \leq\left|P_{i j}^{t}\right| \leq P_{i j}^{\max }
$$

where $P_{i j}^{t}$ is the power between nodes $i$ and $j, P_{i j}^{\min }$ is the minimum power between nodes $i$ and $j$ and $P_{i j}^{\max }$ is the maximum power between nodes $i$ and $j$.

$$
V_{i}^{\min } \leq V_{i}^{t} \leq V_{i}^{\max }
$$

where $V_{i}^{t}$ is the voltage at node $i$ and instant $t$, whereas $V_{i}^{\min }$ e $V_{i}^{\max }$ are the minimum and maximum voltage limits at node $i$, respectively.

$$
P_{\text {load }}^{t}+P_{\text {charge }}^{t}+P_{\text {sell }}^{t}=P_{\text {pv }}^{t}+P_{\text {discharge }}^{t}+P_{\text {purchase }}^{t}
$$

where $P_{\text {load }}^{t}$ is the load power, $P_{\text {charge }}^{t}$ is the power charged by batteries, $P_{\text {sell }}^{t}$ is the power sold for the distribution grid, $P_{p v}^{t}$ is the power provided by photovoltaics, $P_{\text {discharge }}^{t}$ is the power discharged by batteries and $P_{\text {purchase }}^{t}$ is the power purchased from the distribution grid. All the values are for instant $\mathrm{t}$.

$$
0 \leq s t_{\text {charge }}^{t}+s t_{\text {discharge }}^{t} \leq 1
$$

where $s t_{\text {charge }}^{t}$ is the sign of battery charge and $s t_{\text {discharge }}^{t}$ is the sign of battery discharge. The sum of the values cannot be greater than 1 since the battery does not perform the actions simultaneously.

$$
0 \leq P_{\text {charge }}^{t} \leq s t_{\text {charge }}^{t} . P_{\text {chargemax }}
$$

where $P_{\text {charge }}^{t}$ is the power charged by the battery, $s t_{\text {charge }}^{t}$ is the sign of battery charge at instant $t$ ( 0 for no, 1 for yes) and $P_{\text {chargemax }}$ is the maximum power charged by the battery.

$$
0 \leq P_{\text {discharge }}^{t} \leq s t_{\text {discharge }}^{t} . P_{\text {dischargemax }}
$$

where $P_{\text {discharge }}^{t}$ is the power discharged by the battery, $s t_{\text {discharge }}^{t}$ is the sign of battery discharge at instant $t$ ( 0 for no, 1 for yes) and $P_{\text {dischargemax }}$ is the maximum power discharged by the battery.

$$
S O C_{\min } \leq S O C^{t} \leq S O C_{\max }
$$

where $S O C_{\min }$ is the minimum battery state of charge, $S O C^{t}$ is the state of charge at instant $t$ and $S O C_{\max }$ é is the maximum battery state of charge.

$$
S O C^{t+1} \cdot C_{E S S}=S O C^{t} \cdot C_{E S S}+\eta_{\text {charge }} \cdot P_{\text {charge }} \cdot \Delta t-\frac{1}{\eta_{\text {discharge }}} \cdot P_{\text {discharge }} . \Delta t
$$

where $C_{E S S}$ is the battery capacity, $\eta_{\text {charge }}$ is the battery charge efficiency, $\eta_{\text {discharge }}$ is the battery discharge efficiency, $\Delta t$ is a rational number that represents the period between $t$ and $t+1$. In this case $\Delta t=1$.

The multi-objective function can be solved by NSGA-II (Non-dominated Sorting Genetic Algorithm II) [810]. There are differences between single-objective and multi-objective optimization modeling. Singleobjective optimization combines and solves several competing objectives into one global objective function. However, the result may not represent reality as one or more objectives could influence the process. On the 
other hand, multi-objective optimization considers each objective as an objective function and solves them separately [18]. Moreover, single-objective optimization operates in only one dimension while multi-objective optimization is characterized by the existence of a multidimensional space through the objective function [19]. Both designs have countless sets of solutions, although single-objective optimization problems can have a unique solution. At last, both are subject to restrictions which are conditions to be met to enable a certain solution to be feasible [20].

\section{MICROGRIDS INTEGRATION IN DISTRIBUTION NETWORKS}

Relevant research has been developed using optimal power flow (OPF) models to improve both the grid operation itself and the operation of a microgrid integrated to it. However, there is a lack of studies that carry out an optimization by modelling with detail the entire grid with active elements with the microgrids connected to it. In [10], the authors developed an OPF that optimizes a microgrid operation. The main objective is to minimize the price of energy exchange from the microgrid with the main grid, using a multi-period programming and recursive search to determine the microgrid operation parameters. [11] proposed a nonlinear alternating current OPF (ACOPF) model, in which the battery energy storage system is defined to mitigate the fluctuation of a renewable energy generation aiming to determine minimum cost of generation, the storage level change, and the power exchanged with the utility grid. In this case, the ACOPF was solved using the general algebraic modeling system (GAMS) with the interior-point method.

In the present section, an optimal power flow is modeled for a distribution grid considering the representation of a power grid as a whole (not an equivalent circuit) and active elements as distributed generation, storage system, flexible loads, and microgrids. A microgrid can be modeled from its point of common coupling (PCC) with the main grid as a flexible load bus, capable of inserting or absorbing energy to the grid over time.

OPF is an optimization problem in which the objective function defines the grid characteristics that must be optimized and some constraint related to grid operation limits. The proposed OPF modelis solved by the primal-dual interior-point method, with good performance when applied for nonlinear problems.

The proposed methodology minimizes costs of power losses, the costs of operation, costs of battery degradation, and the flexible loads' deviations. The first part that composes the multi-objective function is presented in equation (16), which is the criterion that minimizes the power losses (flosses).

$$
\text { flosses }=\boldsymbol{u}^{\boldsymbol{t}} \text {. }(\boldsymbol{P g}+\text { Pgsolar }- \text { Pd }- \text { Pgld }- \text { Pbat }) \text {. cost }
$$

where $\mathbf{u}$ is a unit vector, Pg is the vector with the power provided by the substation [MW], Pgsolar is the vector with total power provided by the distributed generation sources [MW], Pd is the vector with the total load of the system [MW] and cost represent the energy cost function, based on white tariff values of Brazil [21]; Pbat is the vector with the power injected or absorbed by the battery system and Pgld is the vector with the flexible load values. All the vectors (Pg, Pgsolar, Pd, Pgld, and Pbat) include values referring to 24 hours of planning.

The portion related to the operational costs (foper) is presented in equation (17), which corresponds to the power utility costs to provide power to the grid.

$$
\text { foper }=\mathbf{c}(\mathrm{Pg})
$$

where $\mathbf{c}(\mathbf{P g})$ corresponds to the cost function of power provisioned by the utility (Pg. $\mathbf{c o s t})$. For this end,the white tariff [21] is considered in monetary units (m.u.). It presents higher prices during the peak time (6 p.m. to 9 p.m.) due to grid congestion..

For the storage system degradation cost, a battery system is considered and the degradation cost [22] can be defined as presented in equation (18). The cost $_{\text {ess }}$ represents the price of the battery system, in (m.u.), the DoD is the depth-of-discharge [\%] according to the battery operation, cycles corresponds to the number of life-cycles that this battery system can perform with the DoD specified and Ebat is the total energy storage capacity of the battery [MWh]. Considering a lithium-ion battery, the price of a $2 \mathrm{MWh} / 1 \mathrm{MW}$ system is around 12 million monetary units. For this technology is possible to consider a DoD of $70 \%$ that will entail a life cycle of 4000 cycles (complete charge and discharge) [23].

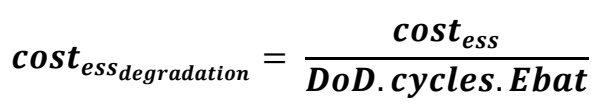


Thus, to obtain the maximum return on investment when purchasing the storage system, the cost of degradation ( e $_{\text {es }} \boldsymbol{s}_{\text {cost }}$ ) should be minimized using the equation (19).

$$
\text { fess }_{\text {cost }}={\text { Pbat. } \text { cost }_{\text {ess }} \text { degradation }}
$$

Finally, the last criterion aims to reduce the deviation of the load management done by the utility (Pdgld) concerning the loads expected by the flexible loads (Pdgld_expected). So, the expected loads of the flexible loads or microgrid integrated with the power grid must be changed if the electricals restrictions are reached, but in a way that the main grid interferes as little as possible in the planning of injection/energy consumption of the microgrid. Such interference no matter how small, directly impacts the optimization problem of a single MG presented in the second section, as it imposes additional restrictions on the variables $P_{\text {purchase }}^{i, t}$ and $P_{\text {sell }}^{i, t}$ from the objective function (1). Thus, the load management deviation objective function is modeled by (20).

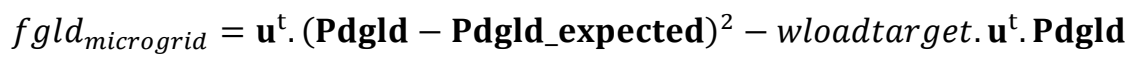

where Pdgld_expected is the vector with the load values expected by the flexible loads (microgrid); Pdgld is the vector with the load values optimized by the OPF in a way to satisfy the electrical restrictions; wloadtarget corresponds to the weight that considers the importance of keeping the expected total load maximized.

Therefore, it is possible to gather all the criteria, composing a multi-objective function presented in equation (21).

$$
\text { O. F }=\text { wp.flosses }+ \text { wc.foper }+ \text { wbat. fess } \text { cost }+ \text { wgd.fgld } \text { microgrid }
$$

where $w p, w c, w b a t$ and $w g d$ are weights related to each one of the functions. The weights values should be adjusted to allow the best performance of the optimization presenting striking results as expected.

The complete OPF formulation, objective function, and constraint equations are presented at equations (22) to (27), in which values are referred to each of the 24 hours of planning. The equality equations represent the active and reactive power balance (equations (22) and (23), respectively). Moreover, inequality expressions represent the operational limits. Equation (24) is related to the voltage operational limits, equation (25) to voltage regulator tap levels, equations (26) and (27) the limits of power and energy of the battery energy storage system.

$$
\text { O.F }=\text { wp.flosses }+ \text { wc.foper }+ \text { wbat. } \text { fess }_{\text {cost }}+\text { wgd.fgld } \text { microgrid }
$$

s.t.

$$
\begin{gathered}
\text { Pg }+ \text { Pgsolar }- \text { Pd }- \text { Pdgld }- \text { Pbat }=\mathbf{P} \\
\text { Qg }+ \text { Qgsolar }- \text { Qd }- \text { Pdgld } \tan (\operatorname{acos}(\boldsymbol{p} f))-\text { Pbat } \tan (\operatorname{acos}(\boldsymbol{p} f))=Q \\
\operatorname{Vmin} \leq \mathrm{V} \leq \text { Vmax } \\
\operatorname{amin} \leq \mathbf{a} \leq \text { amax } \\
- \text { Pbatmax } \leq \text { Pbat } \leq \text { Pbatmax } \\
\text { Ebatmin } \leq \text { Ebat } \leq \text { Ebatmax }
\end{gathered}
$$

where $\boldsymbol{p} \boldsymbol{f}$ is the power factor of the battery system and flexible loads, $\mathbf{a}$ is the vector of tap position of voltage regulators.

For the analysis, a 90-bus test feeder (Figure 1) was considered. It is based on the 69-bus system from [24], plus 20 low voltage buses [25] and 16 distributed equivalent solar generators [26]. A battery system was appended to the substation bus [23]. Flexible loads and microgrids were inserted at buses 27, 36, 48, 64 , and 66.

In order to carry out the analysis and comparison of system behavior and impacts on power grid optimization, different scenarios were considered, together with their results (Table 1). 


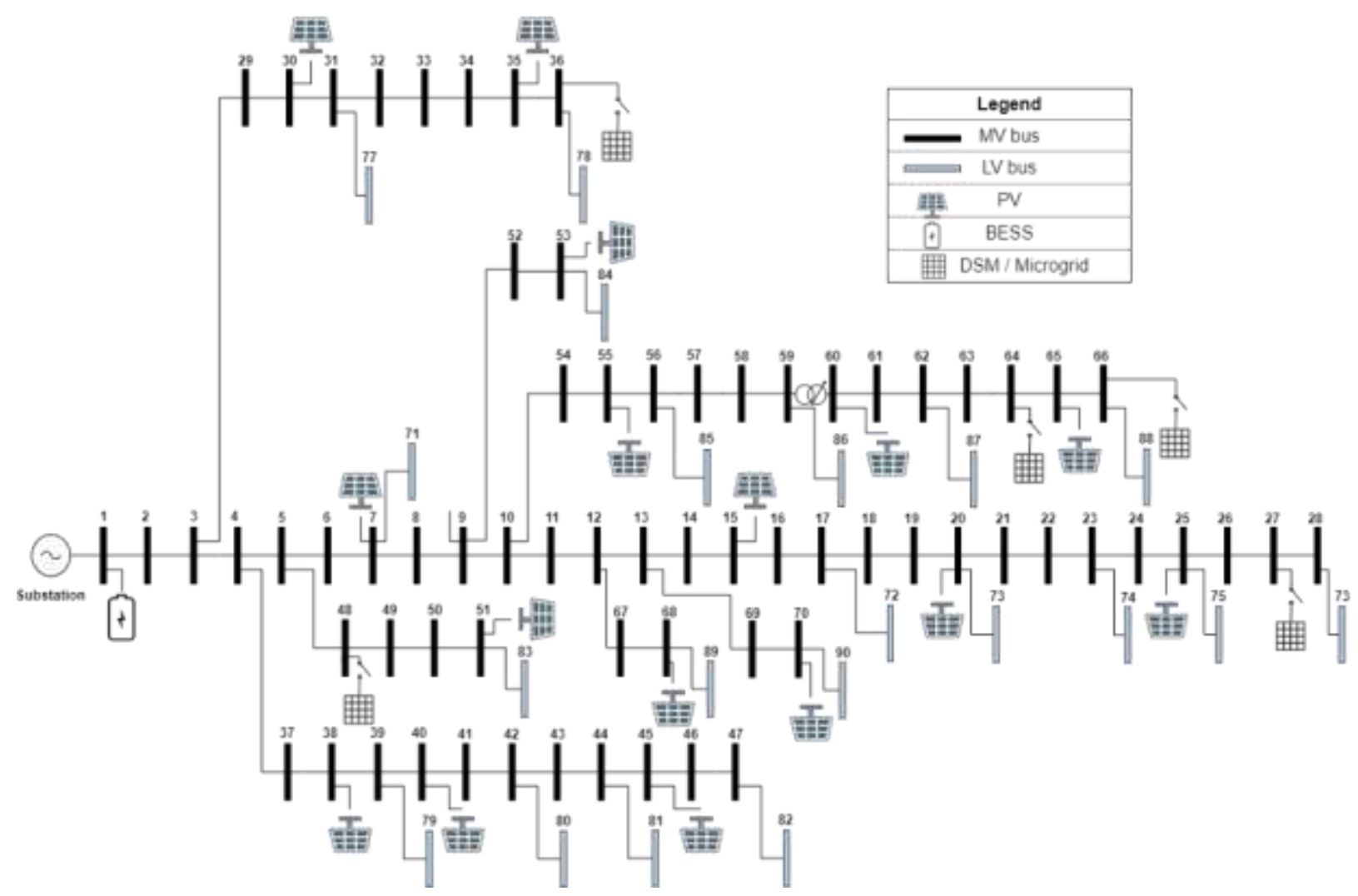

Figure 1. A schematic diagram of the modeled and simulated 90-bus test circuit.

Table 1. Simulation scenarios and results.

\begin{tabular}{|c|c|c|c|}
\hline & Description & $\begin{array}{l}\text { Losses } \\
\text { (MWh) }\end{array}$ & $\begin{array}{l}\text { Operational } \\
\text { Cost (m.u.) }\end{array}$ \\
\hline $\begin{array}{l}\text { Scenario } 1 \\
\text { (base) }\end{array}$ & $\begin{array}{l}\text { Distribution grid just with distributed generation (solar } \\
\text { photovoltaic) }\end{array}$ & 2.52 & $35,361.97$ \\
\hline Scenario 2 & $\begin{array}{l}\text { Scenario } 1+\text { connection of a storage system (battery) } \\
\text { at the substation bus. }\end{array}$ & 2.97 & $34,895.16$ \\
\hline Scenario 3 & $\begin{array}{l}\text { Scenario } 1+\text { increase on power demand + demand- } \\
\text { side management (DSM) for flexible loads. The flexible } \\
\text { load operates as a microgrid, which sometimes will be } \\
\text { a load, or a generator or can be disconnected from the } \\
\text { main grid }\end{array}$ & $\begin{array}{r}\text { Without } \\
\text { DSM: } 3.3 \\
\text { With DSM: } \\
3 \\
\text { With } \\
\text { MG:2.19 }\end{array}$ & $\begin{array}{r}\text { Without DSM: } \\
\text { 39,424.61 } \\
\text { With DSM: } \\
\text { 38,125.76 } \\
\text { With MG: } \\
\text { 32,397.90 }\end{array}$ \\
\hline
\end{tabular}

When the battery system is considered (Scenario 2), the total energy loss increased due to the charging period of the battery system, which happens during the solar generation, and thus, more power needs to flow to charge the storage. The operational costs reduce since the battery system discharges during the peak time, contributing to supply the peak load and thus reducing the amount of power that should be provided by the substation at a high price.

The third scenario, however, is more complex, because it is necessary to adjust the weights of the objective function balancing the relationship between cost and flexible load. By trying to minimize the cost, the methodology tends to reduce the load. For this analysis, the power of the buses 27, 36, 48, 64, and 66 are increased and without the DSM strategy, undervoltage levels will happen in some circuit buses (for example, the bus 85 , located upstream of the voltage regulator, presents voltage levels equal to 0.92 p.u.). Nonetheless, when flexible loads are considered in those buses, it is possible to reduce the load demand, mainly during peak time, contributing to keeping all the buses inside of voltage limits. Figure 2(a) presents the active power balance at the substation bus, in which is possible to visualize the complementarity between the power provided by the substation (green color) and the solar generation (red color), as well the load 
reduction during the peak time (yellow color). It represents $14 \%$ of reduction when compared with the scenarios without demand-side management.

At last, consider that the flexible loads are now connected microgrids. The difference consists that in some periods the microgrid injects power into the main grid (grey color). The microgrid load profile operates as a load during the night and the first hours of the day ( 0 to 7 a.m.), is disconnected from the main grid during the solar generation period ( 8 a.m to 5 p.m.), during the peak load time the microgrid provides power to the grid (6 to 9 p.m.) and at least, from 10 to 11 p.m. the microgrids operates as load. In Figure 2(b), it is possible to visualize that when the microgrid is operating as a load, the total demand of the microgrid cannot be supplied by the grid while respecting the operational limits, resulting in a load reduction of $28 \%$ (yellow color). The operational costs are reduced in $1 \%$ when compared with the scenario just with demand-side management and 8\% in comparison with scenario 1.

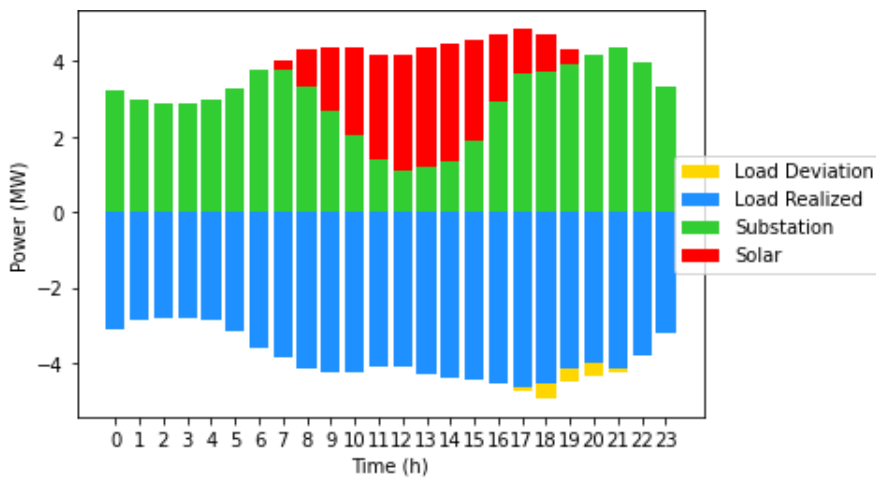

(a)

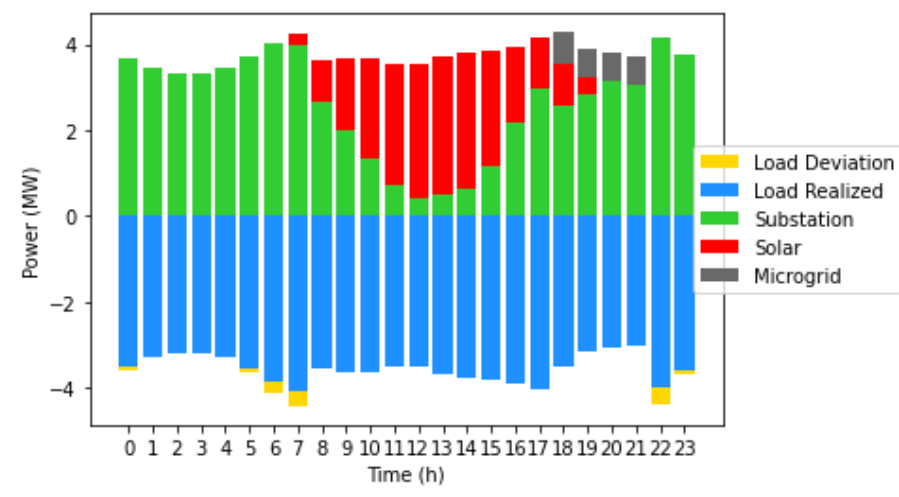

(b)

Figure 2. Active power balance at the substation bus with (a) demand-side management (b) demand-side management and microgrid integration.

From the results is possible to understand the optimization realized by the OPF, considering multiple objectives and considering the operational constraints of the grid. When the microgrid is integrated into the main grid, the total demand of the microgrid could not be supplied by the grid, due to voltage and power flow boundaries in some periods, resulting in load reduction. In practical aspects, the utility OPF results would inform the microgrid control to try to manage the total demand in a given way that the power utility and the microgrid operation could happen in the best optimal for both systems.

\section{MULTI-MICROGRIDS OPTIMIZATION}

When a group of multiple MGs can be operated and controlled in a coordinated manner, taking advantage of electrical connections among microgrids and between MGs and the main grid, such a group has been called a multi-microgrid (MMG) system [13], or microgrid cluster [27], or even networked microgrid [4]. Figure 3 shows a block diagram of a distribution network with multi-microgrids. The advantages of an MMG go beyond the well-known advantages of individual microgrids since the association of multiple microgrids can result in additional benefits. Microgrids belonging to an MMG can provide support to each other in the occurrence of contingencies, as in the $M M G_{B}$ shown in Figure 3 . Also, they can improve the efficiency of a distribution system and increase its reliability and resilience $[4,13,27]$.

In an MMG, each element (MG) can have operational independence as it must be able to operate individually in a group dismemberment event. Also, the elements of a group can operate for their purposes, which can be independent of those in the group. However, the operation of the group (MMG) has to be collaborative as its elements must have at least one common goal, as suggested for the MGs belonging to $M M G_{A}$ and $M M G_{B}$ shown in Figure 3. As a consequence, an MMG fits the definition of system-of-systems (SoS) presented in [28] and verified in [13]. Also, an MG in a distribution network with multiple MGs is not necessarily participating in an MMG group, as the $M G-c$ shown in Figure 3. Finally, the operation of an MMG requires coordination with the DSO and can be performed by an energy management system (EMS), whose real-time decisions can be made automatically and free of human intervention. 


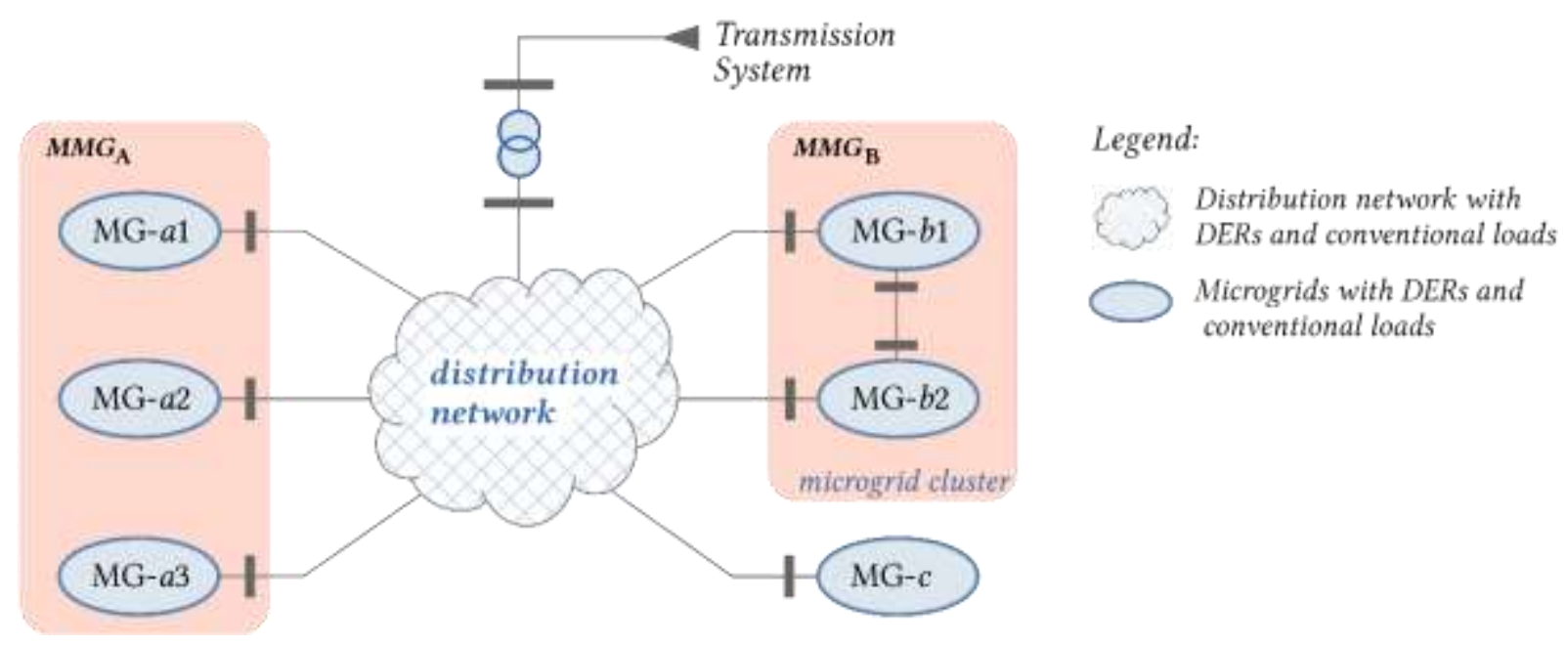

Figure 3. Block diagram of a distribution network with multi-microgrids.

MMG optimization has been investigated in several studies in the literature [12-14]. Authors in [13] propose an SoS approach to modeling the energy management of an MMG system. To reduce the operating costs of a distribution network, an optimization problem considering uncertainties in PV and wind (WT) generation was presented in the article. Two optimization problems are solved iteratively in the method proposed by the authors. In the former, a Distribution Management System (DMS) treats each MG with a single entity and aims to reduce both the cost of energy exchange between pairs of MGs and between a distribution network and an MG. In the latter, each MG central controller (MGCC) has the objective of reducing both costs with energy resources (PV, WT, diesel, ESS, load shedding) and with the purchase of energy from other MG and the distribution network. Authors conclude that the collaborative operation between MG has a lower operating cost compared to an independent operation. In [12], the authors formulate the problem of operating an MMG system as a unit commitment problem. In that work, a coordinated operation approach allows an MG to exchange energy with both the DN and other MGs. Authors consider a scenario where several homes containing rooftop PVs, controllable loads, conventional loads, and electric vehicles as storage units are connected to a DN. Thus, each house is an MG, and the complete system is called residential MMG. As in [13], authors in [12] also conclude that operating independently, exchanging energy only with DN, may be more costly than operating collaboratively. In the study presented in [14], the authors propose a decentralized energy management framework for MMG operation. Dispatchable distributed generation, such as microturbine generators, controllable loads, energy storage, PV and WT generation, and conventional loads were considered in the model. Also, the model considers DER connected across the DN outside the MG. The distribution system and MG are operated by different entities, which, although having their objectives, also have the common purpose of reducing the overall cost of the DN operation. Tests were performed on the IEEE 33-bus model modified with three MG. The results show the validation of the algorithm and the consequent optimization of MMG costs.

Optimizing the operation of a single MG implies determining the optimal operating point for each internal asset during a day of operation, as presented in the second section. On the other hand, optimizing an MMG involves determining the optimal operating point for each MG in the distribution network. In the first case, the main interest is to reduce MG operating and energy costs. In the second case, in addition to the concern with energy costs and expenses in general, there is also a preoccupation with violations of operational limits throughout the distribution network, which requires the calculation of the power flow in the distribution network with MMG, as discussed in the third section. Thus, the results can be used as constraints on the optimization problem of each MG. Consequently, conflicts of interest between the distribution system operator and the microgrids operators may arise. For example, an action to perform an optimum power flow (OPF) to reduce losses in an MMG area can limit the power flow at the point of common coupling of an MG and, as a result, increase its energy costs. Therefore, performing an MMG optimization problem requires an interaction between the optimization algorithms of the DSO (as in equation (21), for example) and MGCC to achieve the best solution for the group that is suitable for all stakeholders, as presented in Figure 4.

Figure 4 illustrates a block diagram of an interactive methodology used to solve the MMG optimization problem. Although objective functions may change with the problem approach (e.g., OPF or minimizing operating costs) and the available distributed energy resources, this methodology may remain, as it can 
address conflicts of interest among stakeholders. According to the diagram in the figure, for each step of the MMG optimization algorithm, each MGCC solves a complete optimization problem. After that, the MGCC optimal results are sent to the MMG algorithm for the next step. This procedure continues until the MMG algorithm reaches convergence. A similar methodology was used, for example, in [13] and [12]. The algorithm begins with a power flow analysis (PFA) of the MMG distribution network area. The result of the PFA is an initial feasible vector $\mathbf{z}$ of active powers between MGs and between MGs and the main grid that meets the operational and contractual restrictions of the distribution network and the MMG. Then, the DSO passes on that information to each MGCC and waits for the optimal cost vector $\boldsymbol{v}^{*}(\mathbf{z})$ to continue its optimization process. Upon receiving the vector $\mathbf{z}$, each MGCC applies it in the MG power balance constraint to perform the optimization. Next, the optimal cost vector $\boldsymbol{v}^{*}(\mathbf{z})$ for the current iteration $k$, obtained by all MGCC, is passed on to the DSO that makes up its first feasible solution to the optimization problem. The procedure ends when the MMG algorithm converges to an optimal value that represents a collaborative optimization of the group.

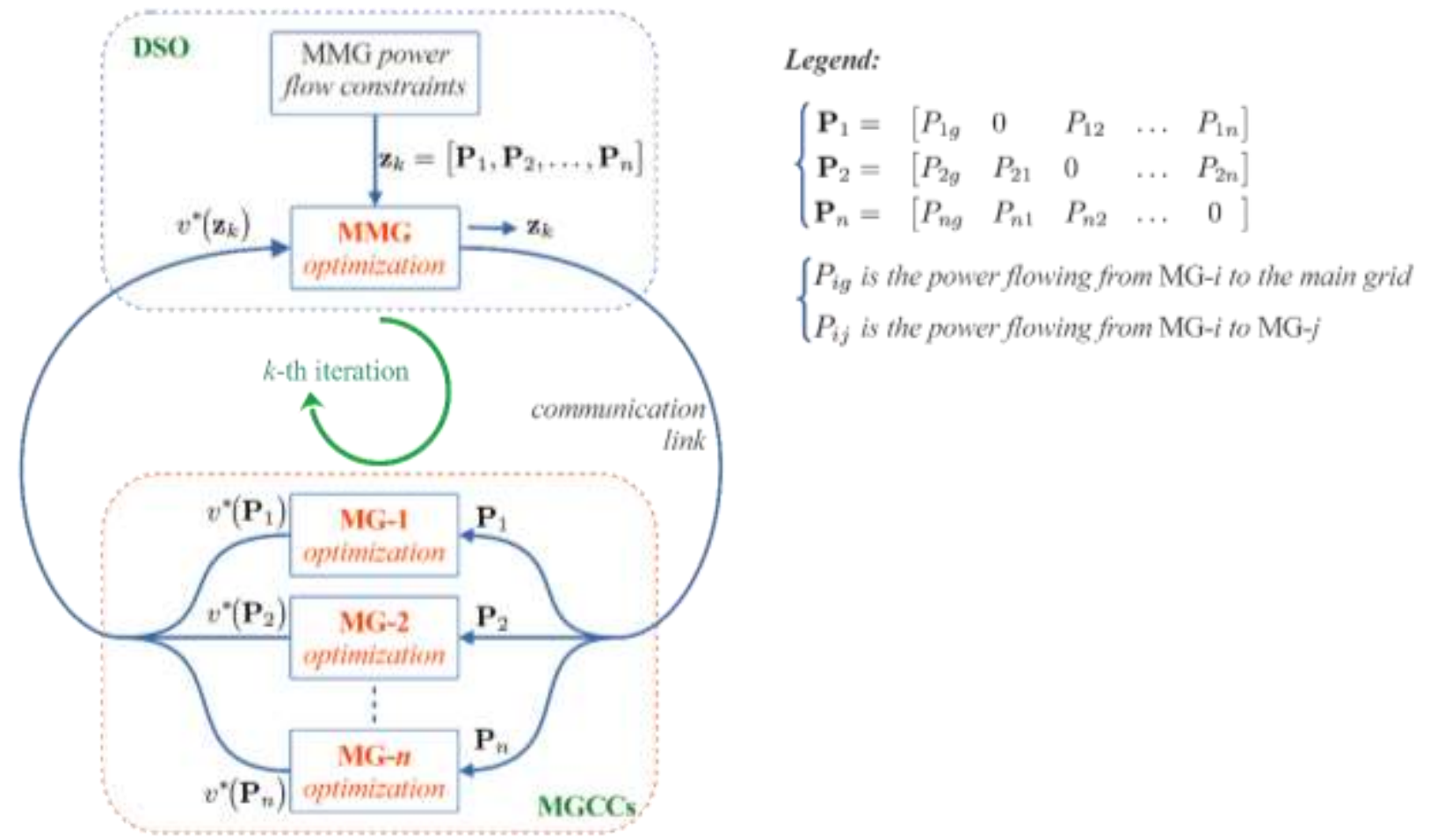

Figure 4. Block diagram of a methodology for MMG optimization.

In the previously mentioned Copel's project [5], there is an incentive offered to energy producers who want to form microgrids in the Copel's distribution network and thus contribute to improving the company's reliability indicators. With such an incentive, the State of Parana may soon have several microgrids in its distribution network. Therefore, the company will be able to apply MMG approaches in those MGs, which may be a relevant action to optimize its distributed energy resources and better serve consumers. On the other hand, it can also be an opportunity for researchers to study and validate MMG architectures in practice.

\section{MICROGRIDS AND DECENTRALIZED ENERGY MARKETS}

As a potential solution for orchestrating the growing amount of decentralized energy resources with the reliable operation of the grid, the market-driven operation of distribution systems has attracted significant research attention in recent years. Fundamentally, transaction-based operation differs from demand response platforms in a way that prosumers are not reacting to price signals, but actively negotiate their energy demands and offers [29].

Under the theoretical framework of microgrid transactive energy system, each connected user (for example a smart home, building, industrial site, storage owner, electric vehicle, etc.) can engage in market trade for energy, individually or clustered in microgrids, negotiating a transaction at the distribution system level [30].

As an alternative to coordinated approaches that orchestrate the response of DERs and MGs by casting the energy management problem as an optimization problem, such as shown in the previous sections, the 
prosumer/microgrid energy trading aims to integrate DERs by establishing decentralized energy markets [31]. The market, if properly designed, works as an energy management system in itself, using traded prices as a key operational parameter. Both the energy demands and surpluses from an MG may be actively traded as long as the operational restrictions imposed by the DSO or by the physical limits of the grid are obeyed.

In a microgrid paradigm, such a market-driven operation can tap into the flexibility of the prosumers to help locally balancing of supply and demand, minimize operational costs for the market participants, minimize the dependency of the network in grid-connected mode and avoid load shedding in islanded mode [32-35].

Based on [36], the mechanisms and enabling technologies that comprise a transactive energy system can be broken down and classified into 5 layers, according to Figure 5.

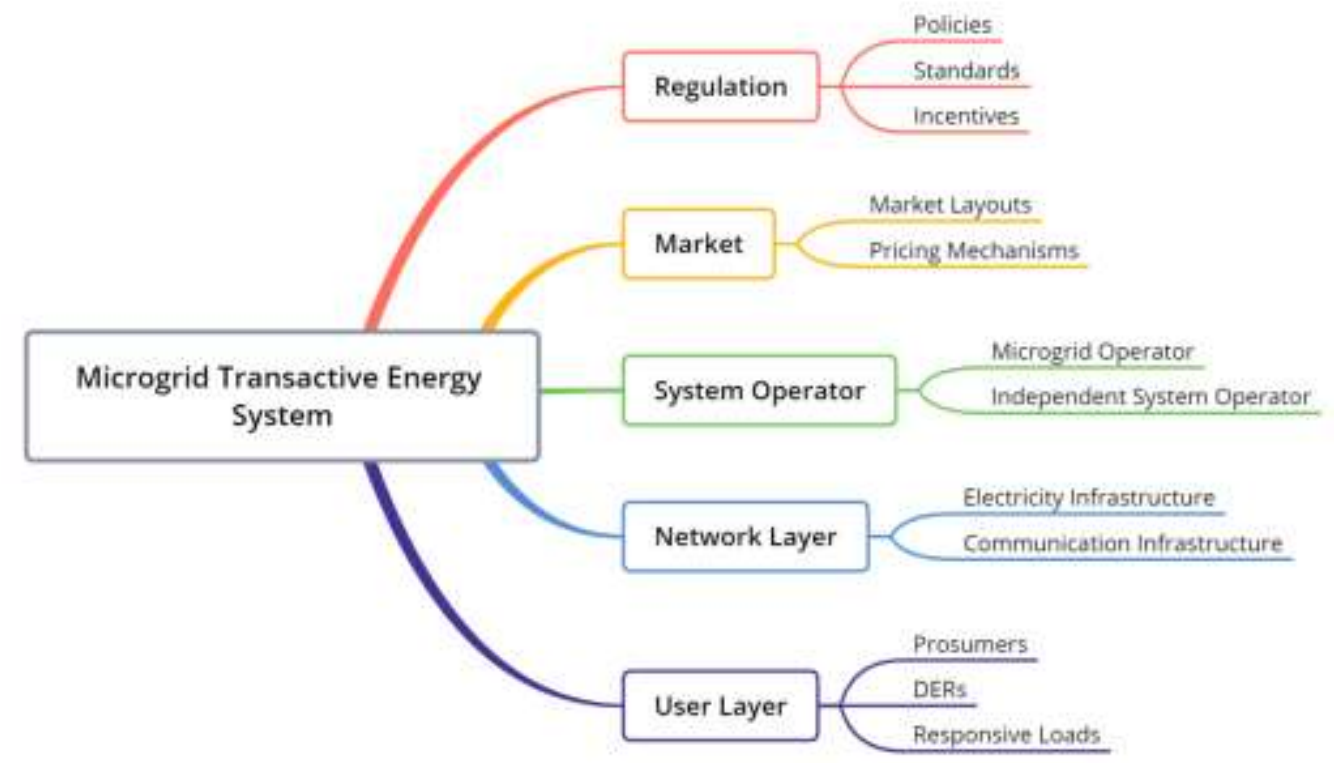

Figure 5. Layer model for microgrid transactive energy system.

The user layer is composed of the participants and their DERs. The most important feature of this layer is prosumer flexibility, provided mainly by storage systems and responsive load devices. This flexibility is considered as a resource, allowing these agents to tune their preferences and own individual objectives with the systems operation objectives employing energy trading. Microgrids play a role in aggregating these agents into communities or energy collectives which potentializes the economic value for the individual participants.

The network layer includes the electricity and communication infrastructure. The transactions and delivery of electricity are inherently linked to this electrical physical system, whose operation reliability is a fundamental trait. The grid architecture and microgrid setups have a profound influence on the market outcomes considering the delivery of traded energy must respect the network constraints. Microgrids in gridconnected mode, for example, are impacted by their congestion limit in their point of common coupling. In islanded mode, on the other hand, the market-based operation must ensure dynamic supply and demand balance.

The communication infrastructure must guarantee a fast and reliable exchange of information between all participants through, among other things, a suitable amount of bandwidth, coverage, and latency. In terms of information systems, one of the main technologies being explored to enable decentralized energy trading is blockchain. Blockchain is seen as particularly promising in the area of peer-to-peer (P2P) trading and decentralized energy management since through blockchain a large number of self-interested actors can be connected and coordinated. In [37], the concept of blockchain-based power microgrids without the need for central intermediates is explored. The study makes a separate analysis of microgrid energy markets and blockchain technology, deriving from this knowledge seven criteria for the efficient operation of the blockchain-based microgrid energy markets. Finally, it shows as a case study the "Brooklyn Microgrid" (BMG) project, considered the world's first real microgrid to operate blockchain-based. The study in [38] proposes an integrated blockchain-based energy management platform is proposed to optimize energy flows in a microgrid whilst implementing a bilateral trading mechanism. The model is implemented on a blockchain network with a smart contract acting as a virtual aggregator. 
The system operator layer is responsible for orchestrating and monitoring the operation of the power system during the trade and delivery of energy. In a transactive energy system, the microgrid energy management system may act as a controller and market operator and may communicate with an independent system operator from the main grid in hierarchical levels. This energy management system can drive the energy trading and operation according to multiple objectives such as the balance of demand and supply, stability, power quality, resiliency, minimizing losses, costs, or maximizing revenues.

In the market layer, different mechanisms and structures can be used to establish transactions between market participants. The layouts can range from a full electricity pool format (Figure 6 ), where the prosumers exchange their bids and offer with a central coordinator that calculates the single system marginal price, to a full bilateral trading paradigm, also known as peer-to-peer, where every market participant negotiates directly with one another. A combination of these two layouts can be set up, with the inclusion of aggregation nodes. These aggregators can have a physical limit in form of microgrids, with clearly defined electrical boundaries, or be cast in a virtual form, such as virtual power plants. The aggregation can provide energy trading in hierarchical levels, from prosumers within a microgrid, prosumers outside the microgrid, and inter-microgrids, for example. These combinations could incentivize local trading and provide scalability to a system with a large number of trading agents. In this layout, the individual interests may be superseded by the interest of the group. For example, the study in [39], where the designed system has more advantage in a collective mode than a solution achieved individually. A similar conclusion was obtained for the MMGs in the fourth section.

In the domain of pricing mechanisms, three main techniques can be distinguished, as based in [40] and shown in Table 2.

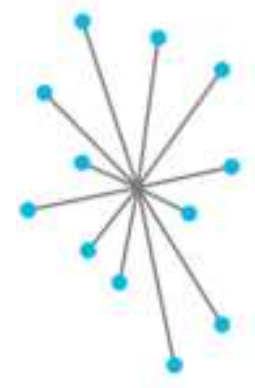

Electricity Pool

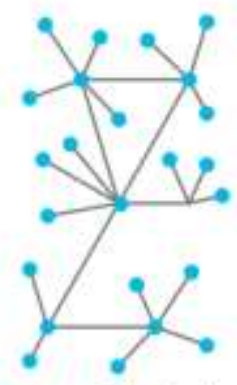

Prosumer Clustering

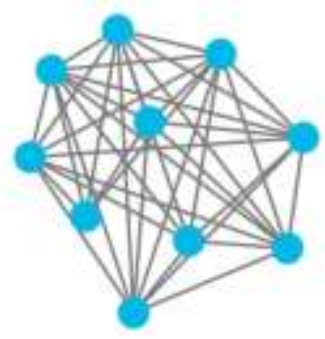

Bilateral Trading Peer-to-peer

Figure 6. Electricity market layouts.

Table 2. Pricing mechanisms classification based in [40].

\section{Game Theory}

Auction Theory

Constrained optimization
Competition or cooperation in an iterative process to deliver a solution that is stable and mutually beneficial Interaction between several sellers and buyers in a stepby-step fashion, like the stock exchange.

Mathematical programming where $\mathrm{P} 2 \mathrm{P}$ trading is cast as an optimization problem.

Finally, the regulation layer has the important task of driving this transition to active and decentralized power systems by integrating the microgrid transactive energy framework to other electrical markets and the bulk power system. The regulatory framework must therefore ensure that the designed markets provide costreflective trading that brings economic benefits to all stakeholders.

The research of microgrid transactive energy systems is quite new, but despite the significant research attention in recent years, there is much to be yet technically solved. A unified model comprising a bigger picture to capture this kind of market-based operation on large scale is missing, simultaneously looking at issues such as network charges, ancillary services, multi-level storage management, contingencies, and forecast errors, etc. For the P2P energy trading to gain acceptance on a larger scale, it will be necessary for 
utilities to model its technical and economical impacts on the electricity networks and work on integrating the layers that compose these systems. In [41], for example, a co-simulation framework is presented to analyze the impacts of local energy markets in the distribution system.

Several pilot projects are being developed worldwide, such as the Brooklyn Microgrid [37], to better understand this market-based operation and to increase interest and awareness between the prosumers. In Brazil, Copel's pilot project [5] must contain a comparison analysis on investment and return from grid reinforcement versus implementation of microgrids to achieve the desired grid reliability. This analysis gives the opportunity to show the potential of market-based operation and its economical benefits for the operator as well as for the community-based prosumers.

\section{CONCLUSION}

This study sought to present a summary of the current research at the State of Parana on the topic of microgrids, with the view of enabling a microgrid-based distribution system infrastructure. It featured a broad view of an MG and its operating environment, including technological framework, planning, optimized operation, integration with active distribution networks, multi-microgrid environment, and market issues. The studies are shown from their overlying relationship, from the optimization of a single MG to the optimization of a multi-microgrid structure connected to the main grid.

The optimization of a single and independent MG was presented in second section. It requires modeling the PV system to estimate the generation capacity for the next day, modeling the storage system to estimate its degradation at each cycle, model the load to estimate MG consumption for the next day, in addition to observing both the operational limits of the assets and those of the distribution network. Such a mathematical programming problem has been solved using Genetic Algorithms.

On the other hand, when analyzing MG in an integrated way with the distribution system as in the third section, it was found that the interests of the distribution system operator are at a level above the interests of an MG. Thus, the optimization of the distribution system as a whole can result in the imposition of operational limits by the DSO for the MG power flow at the point of coupling. In practical cases, this means limits for the $M G$, as presented in the second section, to buy and sell energy.

The growing number of MGs in the distribution network should enable practical cases of operation for a group of MGs with common operating objectives. Although they are integrated with the DN and subject to the restrictions commented on in the third section, such MGs can still constitute an MMG and thus benefit from the optimized group operation, as discussed in the fourth section. Thus, it is possible to conclude from the second, third, and fourth sections that an MG can operate in an integrated manner with the active distribution networks, in an MMG environment, without, however, failing to have its individual operating objectives.

It should be considered that soon, MGs, whether operating independently or participating in an MMG group, may be inserted in decentralized and transaction-based energy markets, as described in the fifth section. It was verified in this section that, although it can be an attractive market for microgrid operators, there are many challenges to be overcome for this type of market to be operational, such as regulatory policies, standards, incentives, price mechanisms, among others.

Finally, due to the public call Copel 001/2020, the State of Parana may soon have an environment with multiple microgrids and will have the opportunity to implement the concepts of MGs and MMGs optimization, decentralized energy markets, and their integration into an active distribution network, as presented in this work.

As a result, these studies contribute in a practical way to the path for enabling a decentralized, microgridbased paradigm of distribution systems. When reviewed together, they allow a more holistic view to research and development of microgrid technology.

Funding: This research received no external funding.

Acknowledgments: Alexandre Rasi Aoki thanks the grant for Technology Development of CNPq-National Council for Scientific and Technological Development within the Ministry of Science, Technology, Innovations, and Communications. The authors also thank the support of CAPES—Brazilian Federal Agency for Support and Evaluation of Graduate Education within the Ministry of Education of Brazil.

Conflicts of Interest: The authors declare no conflict of interest. 


\section{REFERENCES}

1. Ton DT, Smith MA. The U.S. Department of Energy's Microgrid Initiative. Electr J. 2012;25(8):84-94.

2. Marnay C, Chatzivasileiadis S, Abbey C. Microgrid Evolution Roadmap. 2015 Int Symp Smart Electr Distrib Syst Technol. 2015;

3. Hederman WF. IEEE Report to DOE QER on Priority Issues. IEEE Power and Energy Society. Washington, DC; 2014.

4. Alam MN, Chakrabarti S, Ghosh A. Networked Microgrids: State-of-the-Art and Future Perspectives. IEEE Trans Ind Informatics. 2019 Mar;15(3):1238-50.

5. Chamada Pública Copel DIS GD 001/2020 - Compra de Energia Elétrica de Geração Distribuída [Internet]. Curitiba, PR: COPEL; 2020. p. 69. Available from: https://www.copel.com/hpcweb/wp-content/uploads/2020/11/EditalChamada-Publica-DIS-GD001-2020site.pdf

6. Silva VA, Aoki AR, Lambert-Torres G. Optimal Day-Ahead Scheduling of Microgrids with Battery Energy Storage System. Energies. 2020 Oct 5;13(19):5188.

7. Deihimi A, Keshavarz Zahed B, Iravani R. An interactive operation management of a micro-grid with multiple distributed generations using multi-objective uniform water cycle algorithm. Energy. 2016 Jul;106:482-509.

8. Sedighizadeh M, Esmaili M, Jamshidi A, Ghaderi M-H. Stochastic multi-objective economic-environmental energy and reserve scheduling of microgrids considering battery energy storage system. Int J Electr Power Energy Syst. 2019 Mar;106:1-16.

9. Wang L, Li Q, Ding R, Sun M, Wang G. Integrated scheduling of energy supply and demand in microgrids under uncertainty: A robust multi-objective optimization approach. Energy. 2017 Jul;130:1-14.

10. Levron Y, Guerrero JM, Beck Y. Optimal Power Flow in Microgrids With Energy Storage. IEEE Trans Power Syst. 2013 Aug;28(3):3226-34.

11. Maffei A, Meola D, Marafioti G, Palmieri G, lannelli L, Mathisen G, et al. Optimal Power Flow model with energy storage, an extension towards large integration of renewable energy sources. IFAC Proc Vol. 2014;47(3):9456-61.

12. Zhang B, Li Q, Wang L, Feng W. Robust optimization for energy transactions in multi-microgrids under uncertainty. Appl Energy. 2018;217:346-60.

13. Zhao B, Wang X, Lin D, Calvin MM, Morgan JC, Qin R, et al. Energy Management of Multiple Microgrids Based on a System of Systems Architecture. IEEE Trans Power Syst. 2018 Nov;33(6):6410-21.

14. Gao H, Liu J, Wang L, Wei Z. Decentralized Energy Management for Networked Microgrids in Future Distribution Systems. IEEE Trans Power Syst. 2018 Jul;33(4):3599-610.

15. Elsied M, Oukaour A, Gualous H, Hassan R. Energy management and optimization in microgrid system based on green energy. Energy. 2015;

16. Liu Y, Yuen C, UI Hassan N, Huang S, Yu R, Xie S. Electricity Cost Minimization for a Microgrid with Distributed Energy Resource under Different Information Availability. IEEE Trans Ind Electron. 2015;62(4):2571-83.

17. Buzza AL. Modelo de Degradação de Baterias para Operação em Sistemas Isolados com Integração de Fontes de Energia Renováveis. Master Thesis, Federal University of Parana; 2020.

18. Yaman S, Lee C-H. A Comparison of Single- and Multi-Objective Programming Approaches to Problems with Multiple Design Objectives. J Signal Process Syst. 2010 Oct 1;61(1):39-50.

19. Akbari M, Asadi P, Besharati Givi MK, Khodabandehlouie G. Artificial neural network and optimization. In: Advances in Friction-Stir Welding and Processing. Elsevier; 2014. p. 543-99.

20. Coello Coello, Carlos A.; Lamont, Gary B.; Van Veldhuizen DA. Evolutionary Algorithms for Solving Multi-Objective Problems. 2nd ed. Boston, MA: Springer US; 2007. (Genetic and Evolutionary Computation Series).

21. Companhia Paranaense de Energia - COPEL, editor. Tarifa Branca [Internet]. Companhia Paranaense de Energia. [cited 2021 Mar 20]. Available from:

https://www.copel.com/hpcopel/root/nivel2.jsp?endereco=\%2Fhpcopel\%2Findustrial\%2Fpagcopel2.nsf\%2Fdocs \%2FB0CA4C8DF4B62F98832581F00058CCF9

22. Tazvinga H, Zhu B, Xia X. Optimal power flow management for distributed energy resources with batteries. Energy Convers Manag. 2015 Sep;102:104-10.

23. Blasi TM. Planning of Active Distribution Systems Operation with Batteries and Renewable Energy Sources. Master Thesis, Federal University of Parana; 2020.

24. Baran ME, Wu FF. Optimal capacitor placement on radial distribution systems. IEEE Trans Power Deliv. 1989;4(1):725-34.

25. Godoi AA. Allocation of Capacitor Banks in Primary and Secondary Electricity Networks. Master Thesis (In Portuguese), Federal University of Parana; 2009.

26. Lachovicz FJ. Reactive Support Planning for Distribution Networks with High Penetration of Solar Photovoltaic 
Generation. Master Thesis (In Portuguese), Federal University of Parana; 2009.

27. Bullich-Massagué E, Díaz-González F, Aragüés-Peñalba M, Girbau-Llistuella F, Olivella-Rosell P, Sumper A. Microgrid clustering architectures. Appl Energy. 2018 Feb;212:340-61.

28. Maier MW. Architecting principles for systems-of-systems. Syst Eng. 1998;1(4):267-84.

29. Parag Y, Sovacool BK. Electricity market design for the prosumer era. Nat Energy. 2016 Apr 21;1(4):16032.

30. Küster KK, Aoki AR, Lambert-Torres G. Transaction-based operation of electric distribution systems: A review. Int Trans Electr Energy Syst. 2020 Jan 2;30(1).

31. Guerrero J, Gebbran D, Mhanna S, Chapman AC, Verbič G. Towards a transactive energy system for integration of distributed energy resources: Home energy management, distributed optimal power flow, and peer-to-peer energy trading. Renew Sustain Energy Rev. 2020 Oct;132:27.

32. Carli R, Dotoli M. Decentralized control for residential energy management of a smart users microgrid with renewable energy exchange. IEEE/CAA J Autom Sin. 2019 May;6(3):641-56.

33. Esfahani MM, Hariri A, Mohammed OA. A Multiagent-Based Game-Theoretic and Optimization Approach for Market Operation of Multimicrogrid Systems. IEEE Trans Ind Informatics. 2019 Jan;15(1):280-92.

34. An J, Lee M, Yeom S, Hong T. Determining the Peer-to-Peer electricity trading price and strategy for energy prosumers and consumers within a microgrid. Appl Energy. 2020 Mar;261:114335.

35. Long $\mathrm{C}$, Wu J, Zhou Y, Jenkins N. Peer-to-peer energy sharing through a two-stage aggregated battery control in a community Microgrid. Appl Energy. 2018 Sep;226:261-76.

36. Zia MF, Benbouzid M, Elbouchikhi E, Muyeen SM, Techato K, Guerrero JM. Microgrid Transactive Energy: Review, Architectures, Distributed Ledger Technologies, and Market Analysis. IEEE Access. 2020;8:19410-32.

37. Mengelkamp E, Gärttner J, Rock K, Kessler S, Orsini L, Weinhardt C. Designing microgrid energy markets: A case study: The Brooklyn Microgrid. Appl Energy. 2018 Jan;210:870-80.

38. van Leeuwen G, AlSkaif T, Gibescu M, van Sark W. An integrated blockchain-based energy management platform with bilateral trading for microgrid communities. Appl Energy. 2020 Apr;263:114613.

39. Cornélusse B, Savelli I, Paoletti S, Giannitrapani A, Vicino A. A community microgrid architecture with an internal local market. Appl Energy. 2019 May;242:547-60.

40. Tushar W, Saha TK, Yuen C, Smith D, Poor HV. Peer-to-Peer Trading in Electricity Networks: An Overview. IEEE Trans Smart Grid. 2020 Jul;11(4):3185-200.

41. Hayes BP, Thakur S, Breslin JG. Co-simulation of electricity distribution networks and peer to peer energy trading platforms. Int J Electr Power Energy Syst. 2020 Feb;115:105419.

(C) 2021 by the authors. Submitted for possible open access publication under the terms and conditions of the Creative Commons Attribution (CC BY NC) license (https://creativecommons.org/licenses/by-nc/4.0/). 